\title{
Pós-operatório de cirurgias cardíacas: complicações prevalentes em 72 horas*
}

Postoperative of cardiac surgeries: prevalent complications within 72 hours

Postoperatorio de cirugías cardíacas: complicaciones prevalentes dentro de las 72 horas

\section{Danieli Covalski ${ }^{\mathrm{I}}$, Eglon Pauli ${ }^{\mathrm{II}}$, Alane Karen Echer ${ }^{\mathrm{III}}$, Raquel Ribeiro Nogueira ${ }^{\mathrm{IV}}$, Vera Lúcia Fortunato Fortes $v$}

\begin{abstract}
Resumo: Objetivo: identificar complicações ocorridas nas 72 horas iniciais do pós-operatório de cirurgias cardíacas e sua associação com características clínicas e demográficas. Método: estudo transversal, realizado com a análise de prontuários dos pacientes submetidos a cirurgias cardíacas entre janeiro de 2018 a dezembro de 2019 em hospital de grande porte. Resultados: dos 252 prontuários avaliados, houve prevalência de homens, idosos, atendidos pelo Sistema Único de Saúde, com comorbidades e que utilizavam medicamentos contínuos. O tipo de cirurgia prevalente foi de trocas valvares unitárias e 75,8\% dos pacientes apresentaram complicações, sendo as mais frequentes de origem cardíaca. Foram observadas associações entre a presença destas com mediana de idade maior, comorbidades e maior tempo de circulação extracorpórea, além de complicações categóricas com reintervenção e mortalidade em 72 horas e geral. Conclusão: foram identificadas complicações cardíacas, renais, hidroeletrolíticas, pulmonares, hematológicas e neurológicas, estando estas, em alguns casos, associadas a aspectos de maior gravidade.

Descritores: Complicações Pós-Operatórias; Procedimentos Cirúrgicos Cardíacos; Prevalência; Cuidados Críticos; Enfermagem
\end{abstract}

Abstract: Objective: to identify complications in the initial 72 hours after cardiac surgery and their association with clinical and demographic characteristics. Method: this is a cross-sectional study, performed with the analysis

\footnotetext{
${ }^{\text {I }}$ Enfermeira, especialista em Cardiologia pelo programa de Residência Multiprofissional em Cardiologia UPF/HCPF/PMPF, Chapecó, Santa Catarina, Brasil. E-mail: dani_covalski@hotmail.com, ORCID: https://orcid.org/0000-0001-5969-0470

II Enfermeiro, especialista em Cardiologia pelo programa de Residência Multiprofissional em Cardiologia UPF/HCPF/PMPF, Passo Fundo, Rio Grande do Sul, Brasil. E-mail: paulieglon@hotmail.com, ORCID: https://orcid.org/0000-0001-6724-5756

III Enfermeira, especialista em Cardiologia pelo programa de Residência Multiprofissional em Cardiologia UPF/HCPF/PMPF, Chapecó, Santa Catarina, Brasil. E-mail: alane_e@hotmail.com, ORCID: https://orcid.org/0000-0003-4668-4498

IV Enfermeira, especialista em Cardiologia pelo programa de Residência Multiprofissional em Cardiologia UPF/HCPF/PMPF, Chapecó, Santa Catarina, Brasil. E-mail: raaquelnogueeira@hotmail.com, ORCID: https://orcid.org/0000-0001-7860-4865

V Enfermeira, Mestre em Assistência de Enfermagem pela Universidade Federal de Santa Catarina, tutora dos programas de Residência Multiprofissional em Atenção ao Câncer e Cardiologia UPF/HCPF/PMPF, Enfermeira da Escola de Educação Profissional - Hospital São Vicente de Paulo, Passo Fundo, Rio Grande do Sul, Brasil. E-mail: pauloverafortes@gmail.com, ORCID: https://orcid.org/0000-0002-9963-9191

* Trabalho de Conclusão de Residência apresentado ao Programa de Residência Multiprofissional em Cardiologia vinculado a Universidade de Passo Fundo/Hospital de Clínicas de Passo Fundo/Prefeitura Municipal de Passo Fundo, 2020.
} 
of medical records of patients undergoing cardiac surgery between January 2018 and December 2019 in a large hospital. Results: of the 252 medical records evaluated, elderly men were prevalent, assisted by the Unified Health System, with comorbidities, and who used continuous medication. The prevalent type of surgery was single valve replacement and $75.8 \%$ of the patients had complications. The most frequent complication was of cardiac origin. Associations were observed between their presence and higher median age, comorbidities, and longer cardiopulmonary bypass, in addition to categorical complications with reintervention and 72-hour mortality and overall. Conclusion: cardiac, renal, electrolyte, pulmonary, hematological and neurological complications were identified, which in some cases are associated with more serious aspects.

Descriptors: Postoperative Complications; Cardiac Surgical Procedures; Prevalence; Critical Care; Nursing

Resumen: Objetivo: identificar las complicaciones en las primeras 72 horas posteriores a la cirugía cardíaca y su asociación con las características clínicas y demográficas. Método: estudio transversal, realizado con el análisis de historias clínicas de pacientes sometidos a cirugía cardíaca entre enero de 2018 y diciembre de 2019 en un hospital de grande porte. Resultados: de las 252 historias clínicas evaluadas, hubo prevalencia de hombres mayores de edad, atendidos por el Sistema Único de Salud, con comorbilidades y que usaban medicación continua. El tipo de cirugía prevalente fue el reemplazo valvular único y el 75,8\% de los pacientes presentó complicaciones, siendo las más frecuentes de origen cardíaco. Se observaron asociaciones entre su presencia y mayor mediana de edad, comorbilidades y circulación extracorpórea más prolongada, además de complicaciones categóricas con reintervención y mortalidad a las 72 horas y global. Conclusión: se identificaron complicaciones cardíacas, renales, electrolíticas, pulmonares, hematológicas y neurológicas, que en algunos casos se asocian a aspectos más graves.

Descriptores: Complicaciones Posoperatorias; Procedimientos Quirúrgicos Cardíacos; Prevalencia; Cuidados Críticos; Enfermería

\section{Introdução}

Embora haja uma redução gradual da sua incidência, nas últimas décadas, as doenças cardiovasculares (DCV) ainda se constituem a principal causa de mortalidade no Brasil e no mundo, correspondendo a aproximadamente $30 \%$ do total de óbitos registrados no país. ${ }^{1} \mathrm{O}$ desenvolvimento destas doenças está estritamente relacionado a fatores como o sedentarismo, o tabagismo, o uso nocivo de álcool e a má alimentação, e a ocorrência determina um aumento de morbidade e impacto elevado na perda de produtividade dos indivíduos afetados. ${ }^{1-2}$

Atualmente, existem inúmeras possibilidades de tratamentos clínicos voltados a essas condições. Entretanto, quando o benefício terapêutico desses recursos se esgota diante do agravamento de patologias que comprometem de forma significativa o funcionamento cardíaco, torna-se necessário empregar abordagens invasivas, sendo que para muitos indivíduos as cirurgias cardíacas são a opção de tratamento mais recomendada e eficaz. ${ }^{3}$ 
Cirurgias cardíacas são procedimentos de grande porte e alta complexidade, que geram intensas repercussões orgânicas, sejam estas ocasionadas pelas correções realizadas e/ou pelos métodos que as viabilizam, tais como o uso de circulação extracorpórea (CEC). Outrossim, aspectos como idade, sexo, presença de comorbidades, tipo de cirurgia e tempo de permanência hospitalar se somam como fatores que podem influenciar no desenvolvimento de alterações e no pior prognóstico ao paciente. ${ }^{4}$

Em função do estado de instabilidade, o período pós-operatório é considerado como um momento crítico, visto que o organismo humano estará no ápice do enfrentamento e adaptação às mudanças intencionais realizadas durante o procedimento cirúrgico, exigindo assim monitoramento e cuidados intensivos, de forma a controlar respostas ineficazes e promover o equilíbrio dos sistemas. ${ }^{5}$

Apesar do aprimoramento científico e tecnológico observado nos últimos anos, complicações nessa fase da recuperação ainda são frequentes. Estudos nacionais ${ }^{4,6-7}$ identificaram o predomínio de complicações pulmonares, cardíacas, infecciosas, neurológicas e renais nas populações analisadas, tendo como consequência o aumento do tempo de internação e, portanto, do risco associado às infecções hospitalares, eventos adversos e de custos monetários e tecnológicos investidos para sua completa recuperação.

Nesse contexto, implica-se a necessidade de cuidados permanentes de forma a se garantir uma recuperação adequada. Os esforços de monitoramento clínico e hemodinâmico no período pós-operatório devem ser constantes, bem como a tomada de decisão rápida, para que as alterações que venham a ocorrer sejam corrigidas o mais breve possível. ${ }^{8}$

A enfermagem tem papel fundamental na assistência ao paciente nesse período, atuando na vigilância, reconhecimento e intervenção imediata. ${ }^{5-6}$ Desse modo, identificar complicações de cirurgias cardíacas que ocorrem nesse período e os aspectos a essas associadas podem fornecer subsídios para auxiliar os profissionais a atuarem antecipadamente, planejando cuidados e protocolos visando uma recuperação adequada, associada à desospitalização precoce. Favorece, além disso, a construção de indicadores no que concerne à avaliação da assistência 
prestada, propiciando uma melhor gestão dos serviços de saúde. ${ }^{9}$ Mediante o exposto, o objetivo deste trabalho foi identificar complicações ocorridas nas 72 horas iniciais do pós-operatório de cirurgias cardíacas e sua associação com características clínicas e demográficas.

\section{Método}

Estudo transversal, analítico, com abordagem quantitativa, realizado em um hospital de grande porte, localizado no município de Passo Fundo, Rio Grande do Sul (RS), referência para alta complexidade cardiológica regional. A pesquisa foi realizada por meio da análise de prontuários dos pacientes submetidos a cirurgias cardíacas na instituição, no período de janeiro de 2018 a dezembro de 2019, atendidos via Sistema Único de Saúde (SUS), convênios e de forma particular, e encaminhados para Unidade de Terapia Intensiva (UTI) adulto geral após o procedimento. O nosocômio não dispunha de UTI coronariana no momento em que esta pesquisa foi realizada. Foram excluídos registros de pacientes que foram a óbito no período transoperatório ou que realizaram procedimentos, nos quais não foi possível corrigir com sucesso a alteração de origem cardíaca apresentada pelo indivíduo.

De forma a evitar duplicidade de dados, para pacientes que foram submetidos a mais de uma cirurgia cardíaca, na mesma internação, foram considerados os dados referentes ao primeiro procedimento, sendo os subsequentes considerados somente para computar o número de novas intervenções, desde que o procedimento original tivesse sido realizado dentro do período estabelecido para coleta de dados e a correção fosse a esse relacionada.

A coleta de dados ocorreu de maio a agosto de 2020, realizada pelos autores, com a utilização de um instrumento semiestruturado (formulário físico) previamente elaborado para este fim, ao qual eram acrescidas as informações pertinentes com base no objetivo do estudo e, posteriormente, digitadas em um banco de dados no programa Excel ${ }^{\circ}$. Os dados coletados referentes aos antecedentes clínicos, informações cirúrgicas e do pós-operatório foram extraídos de evoluções médicas e de enfermagem, além disso, foram aplicados parâmetros 
numéricos nas seguintes situações do pós-operatório: com relação ao sangramento, considerouse como alteração valor de drenagem igual ou superior a $100 \mathrm{ml} / \mathrm{h},{ }^{10}$ e para avaliação dos eletrólitos foram considerados os seguintes valores de referência de normalidade vigentes na instituição para definir presença ou não de distúrbios: potássio 3,6 a 5,2 mmol/L; cálcio 1,00 a 1,20 mmol/L; sódio 135 a $145 \mathrm{mmol} / \mathrm{L}$, magnésio 1,80 a 2,40 mg/dL e cloro 97 a $107 \mathrm{mmol} / \mathrm{L}$.

Os tipos de cirurgias encontrados foram reunidos, conforme similaridade, de forma a permitir uma análise mais consistente dos dados. Assim, os agrupamentos foram: correções aórticas, correções de malformações do septo cardíaco, Cirurgia de Revascularização do Miocárdio (CRM), CRM + correção de Comunicação Interventricular (CIV), CRM + trocas valvares, CRM + valvoplastias, CRM + correção de dissecção da aorta, dupla troca valvar, pericardiectomia, ressecção de tumor intracardíaco, troca valvar aórtica metálica + troca valvar mitral metálica + correção de Forame Oval Patente (FOP), trocas valvares unitárias, troca valvar aórtica biológica + valvoplastia mitral, tubo aórtico reto + valvoplastia aórtica, valvoplastia mitral + correção de Comunicação Interatrial (CIA) e valvoplastias.

Para caracterização da amostra foram utilizadas estatísticas descritivas, como média, mediana e desvio padrão da variável numérica, além de valores mínimos e máximos. Utilizou-se, também, porcentagem para analisar as variáveis categóricas em termos de frequência. A normalidade dos dados foi analisada pelo teste de Kolmogorov Smirnov. As variáveis numéricas foram comparadas por meio do teste de Mann-Whitney e as variáveis categóricas foram analisadas pelo Qui-Quadrado, ambos considerando $\mathrm{p}<0,05$ para resultados significativos. As análises estatísticas foram realizadas por meio do programa BioEstat 5.0.

Ressalta-se que este estudo respeitou os preceitos éticos da Resolução 466/2012 do Conselho Nacional de Saúde, que rege pesquisas envolvendo seres humanos, e recebeu aprovação do Comitê de Ética em Pesquisa sob o CAAE 30700820.9.0000.5342, parecer 3.996.478, em 29 de abril de 2020. 
Pós-operatório de cirurgias cardíacas: complicações prevalentes em 72 horas $\mid 6$

\section{Resultados}

Foram encontrados 259 registros, sendo 7 excluídos em função da impossibilidade de realização do procedimento proposto ou óbito transoperatório, constituindo uma amostra final de 252 prontuários analisados. Destes, $65,5 \%$ dos pacientes eram do sexo masculino e $34,5 \%$ do sexo feminino, com uma idade média de 63,18 anos $\pm 11,614$. A respeito da origem, 17,1\% residiam no município de Passo Fundo, 18,1\% em municípios pertencentes a 6ª Coordenadoria Regional de Saúde, $62,7 \%$ em outras regiões do RS e 1,6\% eram provenientes do Estado de Santa Catarina. Em relação à assistência, 86,1\% dos pacientes foram atendidos pelo SUS e 13,9\% por meio de convênios ou de forma particular, sendo $1,2 \%$ procedimentos realizados em caráter de urgência e os demais $(98,8 \%)$ de forma eletiva.

Em relação às comorbidades e fatores de risco, 92,9\% pacientes apresentavam-nas, com média de 2,6 $\pm 1,362$. Os principais identificados, assim como outros antecedentes em saúde podem ser observados na Tabela 1. Somente 6,3\% dos pacientes apontaram histórico familiar positivo para doenças cardiovasculares. Quanto às medicações de uso contínuo, destaca-se que não foram encontradas informações a respeito em $20,6 \%$ prontuários, ainda assim, 67,8\% dos pacientes faziam uso contínuo de, pelo menos, um medicamento, com média de 3,9 $\pm 2,935$, e 39,6\% utilizavam quatro ou mais medicamentos.

Tabela 1 - Comorbidades/fatores de risco e antecedentes em saúde mais prevalentes na população estudada. Passo Fundo, RS, 2020.

\begin{tabular}{|c|c|c|}
\hline Comorbidades e fatores de risco & $\mathbf{N}$ & $\%$ \\
\hline $\mathrm{HAS}^{*}$ & 178 & 70,6 \\
\hline Ex-tabagista & 63 & 25,0 \\
\hline Diabetes mellitus & 55 & 21,8 \\
\hline Insuficiência cardíaca & 52 & 20,6 \\
\hline Dislipidemia & 47 & 18,7 \\
\hline Tabagista & 32 & 12,7 \\
\hline Obesidade & 31 & 12,3 \\
\hline Fibrilação ou flutter atrial permanente & 24 & 9,5 \\
\hline Hipotireoidismo ou hipertireoidismo & 21 & 8,3 \\
\hline Depressão & 18 & 7,1 \\
\hline \multicolumn{3}{|l|}{ Antecedentes em saúde } \\
\hline IAM prévio & 30 & 11,9 \\
\hline $\mathrm{ACTP}^{*}$ prévio & 13 & 5,2 \\
\hline
\end{tabular}


$\begin{array}{lll}\text { Cirurgia cardíaca prévia } & 11 & 4,4 \\ \mathrm{AVC}^{\S} \text { prévio } & 10 & 4,0\end{array}$

*HAS: hipertensão arterial sistêmica; ' ${ }^{\dagger}$ IAM: infarto agudo do miocárdio; ${ }^{*}$ ACTP: angioplastia coronariana transluminal percutânea; AVCs: acidente vascular cerebral; "FEVE: fração de ejeção do ventrículo esquerdo.

Foram identificados 29 tipos diferentes de cirurgias, que foram agrupados pelos autores conforme similaridade, de forma a facilitar a análise. Os grupos mais realizados foram de trocas valvares unitárias, CRM e correções aórticas. A listagem completa pode ser observada na Tabela 2. O tempo médio de CEC foi de 96,2 minutos $\pm 30,319$ e de clampeamento aórtico de 64,0 minutos $\pm 23,542$. Dos 252 prontuários estudados, somente $1,2 \%$ realizaram procedimentos sem o uso de CEC. Ademais, 40,9\% pacientes receberam hemoderivados durante o transoperatório.

Tabela 2 - Tipos de cirurgias encontrados na pesquisa. Passo Fundo, RS, 2020.

\begin{tabular}{|c|c|c|}
\hline Tipo de Cirurgia & $\mathbf{N}$ & $\%$ \\
\hline Trocas valvares unitárias & 119 & 47,2 \\
\hline Troca valvar aórtica biológica & 55 & 21,8 \\
\hline Troca valvar aórtica metálica & 33 & 13,1 \\
\hline Troca valvar mitral metálica & 20 & 7,9 \\
\hline Troca valvar mitral biológica & 11 & 4,4 \\
\hline $\mathrm{CRM}^{*}$ & 67 & 26,6 \\
\hline Correções aórticas & 15 & 6,0 \\
\hline Correção de dissecção da aorta & 3 & 1,2 \\
\hline Correção de aneurisma com tubo reto & 2 & 0,8 \\
\hline Correção com tubo valvado & 10 & 4,0 \\
\hline $\mathrm{CRM}^{*}+$ Trocas valvares & 14 & 5,6 \\
\hline $\mathrm{CRM}^{*}+$ Troca valvar aórtica biológica & 7 & 2,8 \\
\hline $\mathrm{CRM}^{*}+$ Troca valvar aórtica metálica & 5 & 2,0 \\
\hline $\mathrm{CRM}^{\star}+$ Troca valvar mitral metálica & 1 & 0,4 \\
\hline $\mathrm{CRM}^{*}+$ Troca valvar mitral biológica & 1 & 0,4 \\
\hline Valvoplastias & 9 & 3,6 \\
\hline Plastia valvar aórtica & 4 & 1,6 \\
\hline Plastia valvar mitral & 4 & 1,6 \\
\hline Plastia valvar tricúspide & 1 & 0,4 \\
\hline Correções de malformações do septo cardíaco & 6 & 2,4 \\
\hline Correção de CIA & 5 & 2,0 \\
\hline Correção de $\mathrm{CIV}^{+}$ & 1 & 0,4 \\
\hline Dupla troca valvar & 5 & 2,0 \\
\hline Troca valvar aórtica metálica + troca valvar mitral metálica & 2 & 0,8 \\
\hline Troca valvar aórtica biológica + troca valvar mitral biológica & 3 & 1,2 \\
\hline
\end{tabular}




$\begin{array}{lll}\text { Troca valvar aórtica biológica + Valvoplastia mitral } & 5 & 2,0 \\ \text { Ressecção de tumor intracardíaco } & 3 & 1,2 \\ \text { CRM }^{*} \text { Valvoplastias } & 2 & 0,8 \\ \quad \text { CRM }^{*} \text { + Valvoplastia aórtica } & 1 & 0,4 \\ \quad \text { CRM }^{*}+\text { Valvoplastia mitral } & 1 & 0,4 \\ \text { Pericardiectomia } & 2 & 0,8 \\ \text { CRM }^{*} \text { + Correção de CIV } & 1 & 0,4 \\ \text { CRM }^{*} \text { Correção de dissecção da aorta } & 1 & 0,4 \\ \text { Valvoplastia mitral + Correção de CIA } & 1 & 0,4 \\ \text { Tubo aórtico reto + Valvoplastia aórtica } & 1 & 0,4 \\ \text { Troca valvar aórtica metálica + Troca valvar mitral metálica + } & 1 & 0,4 \\ \text { Correção de FOP } & & \end{array}$

"CRM: cirurgia de revascularização do miocárdio; 'CIA: comunicação interatrial; "CIV: comunicação interventricular; ${ }^{\S} \mathrm{FOP}$ : forame oval patente.

Em relação ao período pós-operatório, $75,8 \%$ dos pacientes apresentaram complicações dentro das 72 horas iniciais. Destes, $75,9 \%$ tiveram de uma a três, $18,8 \%$ de quatro a seis e $5,2 \%$ apresentaram sete ou mais, sendo dez o número máximo de complicações concomitantes apresentadas por um paciente.

No que se refere ao ranking geral, as complicações mais comuns observadas foram o baixo débito urinário (38,9\%), seguido de arritmias (22,2\%), hipertensão (18,7\%), hipotensão $(17,1 \%)$ e hipocalemia (15,1\%). A descrição das complicações encontradas por ordem de prevalência está apresentada na Tabela 3. Categoricamente, por sistemas, foram mais prevalentes, neste estudo, as complicações cardíacas $(46,0 \%)$, seguidas de complicações renais $(38,9 \%)$, hidroeletrolíticas $(25,8 \%)$, pulmonares (19,0\%), hematológicas $(10,7 \%)$ e neurológicas $(7,9 \%)$.

Entre as complicações cardíacas, as mais frequentes foram arritmias $(22,2 \%)$, além de hipertensão arterial (18,7\%) e hipotensão arterial (17,1\%). A arritmia mais comum encontrada foi a fibrilação atrial (FA) (78,6\%), seguido do bloqueio atrioventricular total (BAVT) $(7,1 \%)$, fibrilação ventricular e taquicardia supraventricular (3,6\% cada). Outros tipos de arritmias que apresentaram uma ocorrência cada (1,8\% respectivamente) foram taquicardia ventricular (TV), flutter atrial, associação de FA e BAVT e associação de FA, BAVT e TV.

Das complicações renais, o baixo débito urinário foi o mais prevalente (38,9\%), seguido de insuficiência renal aguda $(4,0 \%)$ e necessidade de realização de hemodiálise $(0,4 \%)$. Em relação às 
alterações hidroeletrolíticas se pode destacar a hipocalemia $(15,1 \%)$, hipercalemia $(6,0 \%)$ e hipocalcemia $(4,4 \%)$. As complicações pulmonares mais comuns nas 72 horas iniciais do pósoperatório foram o tempo de ventilação mecânica (VM) acima de 48 horas (5,2\%), o derrame pleural $(4,8 \%)$ e a reintubação $(4,4 \%)$.

Ademais, associado à categoria de complicações hematológicas foram encontrados o sangramento $(10,3 \%)$ e trombose venosa profunda (TVP) $(0,4 \%)$. Relativo às complicações neurológicas, o delirium foi mais prevalente $(4,8 \%)$, seguido de crises convulsivas $(2,4 \%)$ e Acidente Vascular Cerebral (AVC) isquêmico (2,0\%).

Tabela 3 - Complicações encontradas no estudo por ordem de prevalência. Passo Fundo, RS, 2020.

\begin{tabular}{|c|c|c|}
\hline Categoria de Complicações & $\mathbf{N}$ & $\%$ \\
\hline \multicolumn{3}{|l|}{ Cardíacas } \\
\hline Arritmias & 56 & 22,2 \\
\hline Hipertensão & 47 & 18,7 \\
\hline Hipotensão & 43 & 17,1 \\
\hline Uso de marca-passo epicárdico & 19 & 7,5 \\
\hline Baixo débito cardíaco & 15 & 6,0 \\
\hline $\mathrm{PCR}^{*}$ & 13 & 5,2 \\
\hline Choque & 9 & 3,6 \\
\hline Pericardite & 7 & 2,8 \\
\hline $\mathrm{IAM}^{+}$ & 6 & 2,4 \\
\hline \multicolumn{3}{|l|}{ Renais } \\
\hline Baixo débito urinário & 98 & 38,9 \\
\hline $\operatorname{IRA}^{*}$ & 10 & 4,0 \\
\hline Hemodiálise & 1 & 0,4 \\
\hline \multicolumn{3}{|l|}{ Hidroeletrolíticas } \\
\hline Hipocalemia & 38 & 15,1 \\
\hline Hipercalemia & 15 & 6,0 \\
\hline Hipocalcemia & 11 & 4,4 \\
\hline Hipernatremia & 2 & 0,8 \\
\hline Hiponatremia & 1 & 0,4 \\
\hline \multicolumn{3}{|l|}{ Pulmonares } \\
\hline Tempo de $\mathrm{VM}^{\S}>48 \mathrm{~h}$ & 13 & 5,2 \\
\hline Derrame pleural & 12 & 4,8 \\
\hline Reintubação & 11 & 4,4 \\
\hline Atelectasia & 8 & 3,2 \\
\hline Hipoxemia & 7 & 2,8 \\
\hline Broncoespasmo & 3 & 1,2 \\
\hline Extubação acidental & 2 & 0,8 \\
\hline
\end{tabular}


Pós-operatório de cirurgias cardíacas: complicações prevalentes em 72 horas $\mid 10$

$\begin{array}{lcc}\text { Pneumonia } & 2 & 0,8 \\ \text { Pneumotórax } & 2 & 0,8 \\ \text { Hematológicas } & & \\ \text { Sangramento } & 26 & 10,3 \\ \text { TVP" } & 1 & 0,4 \\ \text { Neurológicas } & & \\ \text { Delirium } & 12 & 4,8 \\ \text { Crise convulsiva } & 6 & 2,4 \\ \text { AVC" } & 5 & 2,0\end{array}$

"PCR: parada cardiorrespiratória; 'IAM: infarto agudo do miocárdio; 'IRA: insuficiência renal aguda; ${ }^{\text {\&VM: }}$ ventilação mecânica; "TVP: trombose venosa profunda; `AVC: acidente vascular cerebral.

Dos 252 prontuários estudados, 4,8\% necessitaram de reintervenção cardíaca dentro das 72 horas iniciais do pós-operatório, em função de sangramento excessivo (4,0\%) e tamponamento cardíaco $(0,8 \%)$. Houve $4,4 \%$ de óbitos nesse período, e no desfecho geral (compreendendo todo o período de internação), a taxa de mortalidade foi de 9,5\%.

As análises realizadas mostraram que, estatisticamente, pacientes que apresentaram complicações tinham uma mediana de idade maior $(66 \pm 10,7$ para pacientes com complicações e $60 \pm 14,9$ para pacientes sem complicações, $\mathrm{p}=0,001)$, presença de comorbidades $(\mathrm{p}=0,047)$ e maior tempo de CEC (91 minutos \pm 30,8 para pacientes com complicações e 80,5 minutos $\pm 27,6$ para pacientes sem complicações, $\mathrm{p}=0,010)$. As variáveis sexo $(\mathrm{p}=0,643)$ e ter recebido hemoderivados no transoperatório $(\mathrm{p}=0,455)$ não foram significativas.

Com relação às variáveis de complicações, de acordo com os sistemas, foram observadas associações significativas de algumas dessas com as variáveis do tipo de cirurgia, reintervenção em 72 horas, mortalidade em 72 horas e mortalidade geral, conforme visualizado na Tabela 4 .

Tabela 4 - Associações propostas entre as variáveis. Passo Fundo, RS, 2020.

\begin{tabular}{lccc}
\hline Variáveis & $\begin{array}{c}\text { Reintervenção } \\
\text { em 72h }\end{array}$ & $\begin{array}{c}\text { Mortalidade em } \\
\text { 72h }\end{array}$ & $\begin{array}{c}\text { Mortalidade } \\
\text { Geral }\end{array}$ \\
\hline Complicações cardíacas & 0,007 & 0,013 & 0,205 \\
Complicações pulmonares & 0,013 & 1,000 & 0,006 \\
Complicações neurológicas & 0,245 & 0,605 & 0,006 \\
Complicações renais & 0,224 & 0,347 & 0,125 \\
Complicações hematológicas & $<0,001^{*}$ & 0,003 & 0,001
\end{tabular}


Complicações hidroeletrolíticas
Os resultados apresentados se referem a valores de $p .{ }^{*}$ Resultado estatisticamente significativo, porém sem
relevância em função de grupo pequeno de pacientes para o cálculo.

\section{Discussão}

Observou-se, neste estudo, que a maior parte da população submetida às cirurgias cardíacas na instituição dentro do período estudado foi do sexo masculino, com uma média de idade de 63,18 anos $\pm 11,614$, corroborando outras pesquisas encontradas na literatura. ${ }^{3,5-6}$ Resultados semelhantes foram encontrados em outros estudos, ${ }^{7,11}$ com predominância de homens $(68,6 \%$ e 65,0\%) e idade média de 60,6 e 65 anos, respectivamente. O predomínio de idosos converge com o envelhecimento populacional e aumento da expectativa de vida observados globalmente, refletindo também a evolução do manejo das doenças por meio de tratamentos clínicos e procedimentos menos invasivos, que prolongam a chegada de pacientes a estágios de maior risco e necessidade de cirurgia., 5

Entretanto, o envelhecimento implica também na diminuição da resposta orgânica à agressão fisiológica que envolve o procedimento, ficando o idoso mais suscetível a instabilidades do que pacientes mais jovens. ${ }^{12}$ Essa assertiva reforça o encontrado neste trabalho, no qual pacientes que desenvolveram complicações nas 72 horas iniciais do pósoperatório apresentaram uma mediana de idade maior do que os que não as desenvolveram.

São escassos os estudos sobre essa temática, que apresentam prevalência do sexo feminino. ${ }^{13-}$ ${ }^{14}$ Observa-se que as mulheres estão associadas a uma indicação cirúrgica tardia, com consequente aumento da mortalidade hospitalar. ${ }^{15}$ Além disso, a disparidade persistente entre os sexos pode estar relacionada com a maior vulnerabilidade dos homens às doenças crônicas, considerando que do ponto de vista sociocultural existem fatores que influenciam comportamentos de maior resistência masculina aos cuidados relacionados à saúde, potencializando a exposição aos fatores de risco e dificultando aspectos de prevenção e de controle de DCV. ${ }^{16-17}$

Neste estudo, a presença de comorbidades e/ou fatores de risco apresentou associação significativa com a ocorrência de complicações no pós-operatório. Houve prevalência deste 
aspecto para $92,9 \%$ dos pacientes, do qual se destacaram a HAS, o ex-tabagismo, o diabetes mellitus, a insuficiência cardíaca e a dislipidemia. Diretamente associado a este fato, a maioria dos pacientes utilizava medicamentos de uso contínuo, em que mais de 1/3 eram polimedicados, de acordo com a classificação da Organização Mundial da Saúde. ${ }^{18}$

A presença de comorbidades e de fatores de risco associados ao histórico do paciente oferecem risco adicional ao desenvolvimento de complicações no pós-operatório e pior prognóstico à realização do procedimento. ${ }^{2}$ Foram observados achados semelhantes em pesquisa realizada com 200 pacientes submetidos à cirurgia cardíaca em um hospital da região Sudeste do Brasil, no qual 75,5\% dos pacientes eram hipertensos, 30\% diabéticos, 13,6\% tabagistas e 12,2\% possuíam insuficiência cardíaca, havendo somente diferença significativa no número de dislipidêmicos, que constituíram $57 \%$ da amostra. ${ }^{19}$

Outro estudo ${ }^{6}$ com pacientes submetidos a CRM e cirurgia de troca valvar no RS também observou grande número de casos de hipertensão arterial (70,9\%), além de sedentarismo (63,3\%), estresse (57,6\%), ex-etilismo $(54,2)$ e ex-tabagismo $(50,9 \%)$.

Houve prevalência, nesta pesquisa, de cirurgias de trocas valvares unitárias, seguido de CRM e correções aórticas, diferentemente dos demais estudos encontrados na literatura. Em pesquisa9 retrospectiva realizada em São José do Rio Preto, São Paulo, com 2.648 pacientes submetidos a cirurgias cardíacas, foi observada prevalência de $62 \%$ de CRM diante de $38 \%$ de cirurgias de trocas valvares, semelhante ao observado em Minas Gerais, ${ }^{4}$ em que as cirurgias mais realizadas foram CRM (53\%), trocas valvares (18\%) e valvoplastias (14\%). Um aspecto ao qual pode ser atribuído essa discordância reside em distinções regionais dos processos de adoecimento, assim como do acesso dos indivíduos ao sistema de saúde, considerando também que existem diferentes pactuações entre regiões de saúde para alta complexidade na área cardiológica.

A CEC foi utilizada na maioria dos procedimentos observados por este trabalho. Apesar da evolução de procedimentos minimamente invasivos, em grandes centros, nos últimos anos, a 
CEC ainda é largamente empregada em cirurgias de correções cardíacas em todo o mundo, tendo revolucionado a realização desses procedimentos ao preservar as características funcionais cardíacas, ao mesmo tempo, em que garante um campo cirúrgico seguro à equipe. ${ }^{2}$

Não há consenso quanto a uma classificação de tempo ideal para manter o paciente nessa forma de circulação, entretanto, sabe-se que quanto menor o tempo a que o paciente fica submetido à CEC menores são os riscos de injúrias a ele relacionados, fato corroborado por este estudo, visto que uma mediana maior de CEC esteve associada ao surgimento de complicações nas 72 horas iniciais do pós-operatório, aspecto observado também em outra pesquisa. ${ }^{20}$

Isso ocorre em função da indução do organismo à resposta inflamatória sistêmica, causada pela passagem do sangue pela superfície não epitelizada dos circuitos extracorpóreos, causando mudanças dos mecanismos de coagulação, resposta imunológica, além de aumento do tônus venoso, alteração do estado hidroeletrolítico, disfunção miocárdica e pulmonar. 2,17 $^{2}$

As complicações nas 72 horas iniciais do pós-operatório foram observadas em 75,8\% dos pacientes, taxa superior ao identificado em outros estudos, ${ }^{4,2,6}$ nos quais as complicações atingiram em torno de 58\%, $52 \%$ e $21 \%$ dos pacientes. Salienta-se que há variabilidade na ordem de prevalência das categorias de complicações por sistemas nas pesquisas citadas, o que também foi identificada nesta análise.

Foram mais comuns as complicações cardíacas, com destaque para as arritmias (principalmente a FA), a hipertensão arterial e a hipotensão arterial. A ocorrência de FA varia de 10 a $40 \%$ de pacientes submetidos a CRM, chegando a 50\% nas trocas valvares e até $60 \%$ na associação dos dois procedimentos. Supõe-se que os fatores que a desencadeiam estejam relacionados à reação inflamatória do pericárdio e ao excesso de catecolaminas, em função do desequilíbrio do sistema autônomo e, como consequência do seu desenvolvimento, há diminuição do débito cardíaco e se predispõe à ocorrência de eventos tromboembólicos. Em até $90 \%$ dos casos há resolução dessa arritmia dentro de dois meses após a cirurgia realizada. ${ }^{21}$ 
Ademais, essa categoria de complicações foi associada, estatisticamente, à necessidade de reintervenção em 72 horas do pós-operatório e à mortalidade no mesmo período. Em conformidade com esse achado, pesquisadores ${ }^{4}$ identificaram a síndrome do baixo débito cardíaco como a complicação que mais trouxe impacto à mortalidade hospitalar em estudo realizado com 211 pacientes submetidos a cirurgias cardíacas, sendo sua persistência por mais de 24 horas associada aos elevados níveis de mortalidade.

O baixo débito urinário foi uma complicação de relevância, sendo a mais prevalente do estudo, apesar de número reduzido de pacientes terem evoluído desta alteração para a Insuficiência Renal Aguda (IRA). Essa complicação é, frequentemente, observada no pós-operatório de cirurgias cardíacas, podendo estar associada ao uso de CEC e a seu tempo prolongado. ${ }^{5}$ Sua ocorrência pode precipitar modificações hemodinâmicas e endócrinas, sendo considerada como um marcador sensível para a detecção de lesões renais agudas, inclusive, quando comparado aos níveis séricos de creatinina, apesar da IRA nem sempre cursar com este parâmetro. ${ }^{22}$

Com o avanço para estágios de dano renal, prolonga-se a internação hospitalar desses pacientes, consumindo mais recursos tecnológicos e humanos investidos em sua recuperação, assim como determina piora do prognóstico, sendo importante a monitorização desse parâmetro para a implementação de medidas de prevenção e de tratamento precoce. ${ }^{22}$

As alterações de eletrólitos foram, principalmente, relacionadas aos níveis séricos de potássio, cálcio e sódio, havendo poucas divergências entre os valores encontrados neste trabalho e nos descritos por outros estudos., ${ }^{2,4} \mathrm{O}$ desequilíbrio hidroeletrolítico no organismo pode acarretar complicações ao paciente, caso não sejam revertidas e, especialmente, em nível cardíaco, destacando-se a influência dos eletrólitos sódio e potássio, diretamente relacionados à condução elétrica cardíaca e à contração muscular, evidenciando assim a importância da sua vigilância. ${ }^{23}$

As complicações pulmonares também são relativamente comuns nesse período, esporadicamente cursando com distúrbios críticos desde que estabelecidas as medidas preventivas 
necessárias. ${ }^{14}$ Seu desenvolvimento pode ser influenciado pelo período de imobilidade ao qual o paciente é submetido, associado também à retirada tardia de dispositivos que dificultam sua saída do leito. ${ }^{9}$ Nesta pesquisa se observou que as alterações dessa natureza estiveram associadas à necessidade de reintervenção dentro de 72 horas após a cirurgia e a mortalidade geral.

O critério para considerar tempo de VM como complicação foi acima de 48 horas, conforme estudo, ${ }^{4}$ entretanto, são observadas variações desse parâmetro na literatura, com pesquisas considerando como complicação tempo superior a 24 horas ${ }^{9,14}$ e até mesmo tempo superior a 12 horas. $^{3}$ Há relato, ainda, de pacientes submetidos a CRM sem CEC, em que se realizou a extubação ainda na sala cirúrgica, ação posteriormente associada à redução do tempo de permanência na UTI. ${ }^{14}$ Essa prática, contudo, não é observada com frequência nos trabalhos da área e não é rotina na instituição, na qual o estudo foi realizado.

O sangramento foi a principal alteração identificada no grupo de complicações hematológicas, estando estas associadas, significativamente, à mortalidade em 72 horas do pósoperatório e à mortalidade geral. A origem desta complicação pode ser multifatorial e, principalmente, relacionada ao procedimento cirúrgico com o uso de CEC..$^{5}$ O contato prolongado com o circuito extracorpóreo, altas doses de heparina, hipotermia e trauma cirúrgico extenso contribuem para o desenvolvimento de coagulopatias no pós-operatório. 5,24

Segundo autores, ${ }^{24}$ esta é uma complicação que pode atingir até $20 \%$ dos pacientes nesse período, e aproximadamente 5\% destes precisarão de nova intervenção cirúrgica para corrigir essa condição. Apesar da ocorrência de sangramento ter sido menor do que o previsto, este estudo encontrou valores semelhantes ao identificar que $4,8 \%$ dos pacientes necessitaram de reintervenção nas 72 horas iniciais após a cirurgia, majoritariamente em função de sangramento excessivo.

Complicações de origem neurológicas foram menos frequentes, aspecto observado também em outras pesquisas ${ }^{2,5-7}$ e sua manifestação esteve estatisticamente associada à mortalidade geral. O delirium é uma condição que ocorre, usualmente, entre o $2^{\circ}$ e $5^{\circ}$ dia de 
pós-operatório e inclui manifestações agudas e flutuantes de delírio, desorientação e dificuldade na linguagem, com incidência geral de aproximadamente $3 \%$ dos casos, podendo alcançar até $20 \%$ em indivíduos com mais de 60 anos. ${ }^{25}$

Crises convulsivas têm incidência menor, $0,5 \%$ a 3,5\% dos pacientes, e se relacionam às condições de hipoxemia, toxicidade medicamentosa e à lesão cerebral estrutural. ${ }^{5}$ Segundo dados da literatura, o AVC atinge por volta de 1,6\% dos pacientes, semelhante ao observado neste estudo, e tem etiologia relacionada à presença de êmbolos, à hipotensão arterial severa e à fibrilação arterial, sendo mais observada em cirurgias de troca valvar do que em CRM. ${ }^{26}$

Os óbitos dentro de 72 horas do pós-operatório representaram 4,4\% da população da pesquisa, com uma taxa de mortalidade durante todo o período de internação de 9,5\%. Considerando o intervalo de 72 horas, não foram encontrados dados na literatura para comparação de resultados.

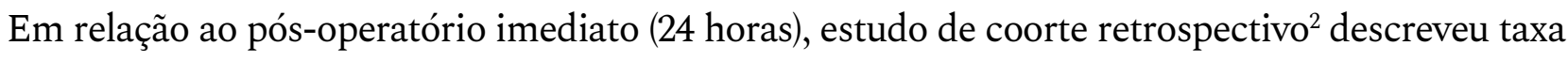
de mortalidade de 4,0\% em uma população de 50 pacientes submetidos a CRM. Já outra pesquisa ${ }^{7}$ observou que $8,7 \%$ dos 103 pacientes submetidos às cirurgias cardíacas foram a óbito dentro de 10 dias da cirurgia. Com valores próximos, após 30 dias do procedimento cirúrgico, foi identificada em pesquisa retrospectiva ${ }^{9}$ uma taxa de mortalidade de $9,1 \%$ de 2.648 pacientes submetidos às cirurgias cardíacas.

Considerando o período de internação total, estudo ${ }^{11}$ realizado em Santa Catarina apontou uma taxa de 5,3\% de mortalidade para pacientes submetidos a CRM, enquanto outros autores ${ }^{19}$ apresentaram taxa idêntica a identificada nesta pesquisa, no qual 9,5\% dos pacientes estudados foram a óbito, considerando uma população total de 200 pacientes. Apesar da discrepância de tempos apresentada se observou semelhança entre a taxa de mortalidade deste trabalho para os demais.

Mediante o exposto, entende-se que os achados observados podem fornecer subsídios que favoreçam o desenvolvimento de ações estratégicas, de forma a melhorar a assistência, por meio de medidas preventivas para os eventos descritos, assim como da sua identificação e intervenção precoce. 
Em suma, tem potencial para contribuir no planejamento de serviços e protocolos direcionados a área, a medida em que são determinados por dados concretos e que refletem a realidade.

A principal limitação desta pesquisa esteve relacionada à origem dos dados, visto que, ao serem consideradas as informações contidas em evoluções médicas e de enfermagem, estão passíveis à subjetividade do profissional em registrar as informações que este considere pertinente.

\section{Conclusão}

Os resultados encontrados no presente estudo apontaram para alta prevalência de complicações nas 72 horas iniciais do pós-operatório de cirurgias cardíacas, estando em alguns casos, inclusive, associados a aspectos de maior gravidade, tal como necessidade de reintervenção operatória e mortalidade. As categorias de complicações mais prevalentes foram as cardíacas (do qual se destacaram as arritmias, a hipertensão e a hipotensão arterial), seguida de renais, hidroeletrolíticas, pulmonares, hematológicas e neurológicas. Em termos de frequência, as complicações aqui identificadas apresentaram semelhanças em relação aos outros estudos da literatura.

A compreensão desses aspectos permite à equipe de enfermagem antever possíveis alterações, que possam acometer os pacientes nesse período, sendo sua identificação precoce fator determinante para garantir um tratamento apropriado e satisfatório. Em um momento de grande instabilidade como é o pós-operatório de cirurgias cardíacas, o conhecimento acerca dos aspectos que prevalecem nesse cenário permite ao profissional aprimorar os cuidados prestados, planejando e desenvolvendo ações apropriadas de forma a assegurar uma recuperação adequada e breve.

Ademais, sugere-se a realização de novos estudos explorando, com mais detalhes, os resultados encontrados e outras indagações por estes provocados, por exemplo, se há diferença no desenvolvimento de complicações entre os diferentes tipos de cirurgias e de acordo com o tempo de CEC. Ainda, recomenda-se que em pesquisas futuras sejam realizados estudos com metodologias que apresentem potencial para determinar causalidade referente às complicações. 


\section{Referências}

1. Malta DC, Teixeira R, Oliveira GMM, Ribeiro ALP. Mortalidade por doenças cardiovasculares segundo o sistema de informação sobre mortalidade e as estimativas do estudo carga global de doenças no Brasil, 2000-2017. Arq Bras Cardiol. 2020;115(2):152-60. doi: 10.36660/abc.20190867

2. Andrade AYT, Tanaka PSL, Poveda VB, Turrini RNT. Complicações no pós-operatório imediato de revascularização do miocárdio. Rev SOBECC. 2019;24(4):224-30. doi: 10.5327/Z1414-4425201900040008

3. Dessotte CAM, Figueiredo ML, Rodrigues HF, Furuya RK, Rossi LA, Dantas RAS. Classificação dos pacientes segundo o risco de complicações e mortalidade após cirurgias cardíacas eletivas. Rev Eletrônica Enferm. 2016;18:1-11. doi: 10.5216/ree.v18.37736

4. Soares GMT, Ferreira DCS, Gonçalves MPC, Alves TGS, David FL, Henriques KMC, et al. Prevalência das principais complicações pós-operatórias em cirurgias cardíacas. Rev Bras Cardiol [Internet]. 2011 [acesso em 2020 nov 01];24(3):139-46. Disponível em: http://sociedades.cardiol.br/socerj/revista/2011_03/a_2011_v24_n03_01prevalencia.pdf

5. Lopes ROP, Castro J, Nogueira CSC, Braga DV, Gomes JR, Silva RC, et al. Complicações do pósoperatório imediato de cirurgia cardíaca eletiva: estudo transversal à luz de Roy. Referência. 2019;serIV(22):23-32. doi: 10.12707/RIV19042

6. Heck LGS, Dallazen F, Cruz DT, Berwanger AS, Winkelmann ER. Análise do período intra e pósoperatório, complicações e mortalidade nas cirurgias de revascularização do miocárdico e de troca valvar. Sci Med [Internet]. 2017 [acesso em 2020 nov 01];27(4):ID28041. Disponível em: https://dialnet.unirioja.es/servlet/articulo?codigo $=6234557$

7. Contrin LM, Beccaria LM, Rodrigues AMS, Werneck AL, Castro GT, Teixeira CV. Complicações pósoperatórias cardiocirúrgicas e tempo de internação. Rev Enferm UPFE On Line. 2018;12(8):2105-12. doi: 10.5205/1981-8963-v12i8a234846p2105-2112-2018

8. Melo FV, Costa MF, Sandes SMS. Diagnósticos de enfermagem no período pós-operatório de cirurgia cardíaca. Rev Enferm UPFE On Line. 2018;12(8):2188-93. doi: 10.5205/1981-8963-v12i8a231671p2188-2193-2018

9. Beccaria LM, Cesarino CB, Werneck AL, Correio NCG, Correio KSS, Correio MNM. Complicações pós-operatórias em pacientes submetidos à cirurgia cardíaca em hospital de ensino. Arq Ciênc Saúde [Internet]. 2015 [acesso em 2020 nov 01]; 22(3):37-41. Disponível em: http://www.cienciasdasaude.famerp.br/index.php/racs/article/view/216/116

10. Minhaj M. Cardiac anesthesia: a problem-based learning approach [Internet]. Oxford: Oxford University Press; 2019 [cited 2021 aug 10]. 408 p. Available from: https://oxfordmedicine.com/view/10.1093/med/9780190884512.001.0001/med-9780190884512.

11. Koerich C, Lanzoni GMM, Erdmann AL. Fatores associados à mortalidade de pacientes submetidos à cirurgia de revascularização do miocárdio. Rev Latinoam Enferm. 2016;24:e2748. doi: 10.1590/1518-8345.0708.2748 
12. Vasconcelos Filho PO, Carmona MJC, Auler Júnior JOC. Peculiaridades no pós-operatório de cirurgia cardíaca no paciente idoso. Rev Bras Anestesiol. 2004;54(5):707-27. doi: 10.1590/S0034-70942004000500014

13. Koerich C, Lanzoni GMM, Meirelles BHS, Baggio MA, Higashi GDC, Erdmann AL. Perfil epidemiológico da população submetida à revascularização cardíaca e acesso ao sistema único de saúde. Cogitare Enferm. 2017;22(3). doi: 10.5380/ce.v22i3.50836

14. Cani KC, Araujo CLP, Karloh M, Alexandrino DFH, Palú M, Rojas DB, et al. Características clínicas de pacientes submetidos à cirurgia de revascularização do miocárdio. ASSOBRAFIR Ciência [Internet]. 2015 [acesso em 2020 nov 01];6(3):43-54. Disponível em: http://www.uel.br/revistas/uel/index.php/rebrafis/article/view/21754/17846

15. Yüksel A, Kan II, Yolgösteren A, Velioğlu Y, Çayır MÇ, Gürbüz O, et al. Are the early postoperative outcomes of coronary artery bypass grafting surgery in elderly women worse compared to men's? Braz J Cardiovasc Surg. 2017;32(3):191-6. doi: 10.21470/1678-9741-2016-0071

16. Mussi FC, Teixeira JRB. Fatores de risco cardiovascular, doenças isquêmicas do coração e masculinidade. Rev Cubana Enferm [Internet]. 2018 [acesso em 2020 nov 01];34(2). Disponível em: http://revenfermeria.sld.cu/index.php/enf/article/view/1613

17. Reis MMR, Lima EFA, Crozeta K, Casagrande RI, Leite FMC, Primo CC. Avaliação do tempo de permanência hospitalar em cirurgia cardíaca em um hospital universitário. Rev Pesq Cuid Fundam. 2020;12:667-75. doi: https://doi.org/10.9789/2175-5361.rpcfo.v12.9158

18. World Health Organization (WHO). Medication without harm: WHO Global Patient Safety Challenge [Internet]. Geneva: World Health Organization; 2017 [cited 2020 Nov 01]. Available from: https://apps.who.int/iris/bitstream/handle/10665/255263/WHO-HIS-SDS-2017.6-eng.pdf?sequence=1

19. Reis MMR, Lima EFA, Casagrande RI, Fioresi M, Leite FMC, Primo CC. Perfil epidemiológico de pacientes submetidos à cirurgia cardíaca. Rev Enferm UPFE On Line. 2019;13(4):1015-22. doi: 10.5205/1981-8963-v13i4a238020p1015-1022-2019

20. Dordetto PR, Pinto GC, Rosa TCSC. Pacientes submetidos à cirurgia cardíaca: caracterização sociodemográfica, perfil clínico-epidemiológico e complicações. Rev Fac Ciênc Méd Sorocaba. 2016;18(3):144-9. doi: 10.5327/Z1984-4840201625868

21. Consolim-Colombo FM, Saraiva JFK, Izar MCO. Tratado de cardiologia Socesp. 4ª ed. Barueri (SP): Manole; 2019.

22. Nascimento MS, Aguiar TC, Silva AVE, Duarte TTP, Magro MCS. Lesão renal aguda no pósoperatório de cirurgia cardíaca. Acta Paul Enferm. 2015;28(4):367-73. doi: 10.1590/1982-0194201500062

23. Freitas LMR, Santos LPCB, Oliveira JB. Circulação extracorpórea e desequilíbrio hidroeletrolítico. J Health NPEPS [Internet]. 2017 [acesso em 08 nov 2020];2(1):285-97. Disponível em: http://docs.bvsalud.org/biblioref/2020/03/1053077/1753-6328-3-pb.pdf

24. Choi YJ, Yoon SZ, Joo BJ, Lee JM, Jeon YS, Lim YJ, et al. A perda sanguínea excessiva no pós-operatório de cirurgia cardíaca pode ser prevista com o sistema de classificação da Sociedade Internacional de Trombose e Hemostasia (ISTH). Rev Bras Anestesiol. 2017;67(5):508-15. doi: 10.1016/j.bjane.2016.12.001 
25. Jin $\mathrm{Z}$, $\mathrm{Hu} \mathrm{J}, \mathrm{Ma} \mathrm{D}$. Postoperative delirium: perioperative assessment, risk reduction, and management. Br J Anaesth. 2020;125(4):492-504. doi: https://doi.org/10.1016/j.bja.2020.06.063

26. Sheth KN, Nourollahzadeh E. Neurologic complications of cardiac and vascular surgery. Handb Clin Neurol. 2017;141:573-92. doi: 10.1016/B978-0-444-63599-0.00031-4

Editora Científica: Tânia Solange Bosi de Souza Magnago

Editora Associada: Rosângela Marion da Silva

Fomento / Agradecimento: Ao Ministério da Saúde, Universidade de Passo Fundo, Hospital de Clínicas de Passo Fundo e Prefeitura Municipal de Passo Fundo pelo fomento às residências multiprofissionais.

\section{Autor correspondente}

Danieli Covalski

E-mail: dani_covalski@hotmail.com

Endereço: Rua Pio XII, n. 46, Apto. 502, Centro, Chapecó, Santa Catarina, Brasil. CEP: 89801-010

\section{Contribuições de Autoria}

\section{1 - Danieli Covalski}

Concepção ou desenho do estudo/pesquisa, análise e/ou interpretação dos dados, revisão final com participação crítica e intelectual no manuscrito.

\section{2 - Eglon Pauli}

Concepção ou desenho do estudo/pesquisa, análise e/ou interpretação dos dados, revisão final com participação crítica e intelectual no manuscrito.

\section{3 - Alane Karen Echer}

Revisão final com participação crítica e intelectual no manuscrito.

\section{4 - Raquel Ribeiro Nogueira}

Revisão final com participação crítica e intelectual no manuscrito.

\section{5 - Vera Lúcia Fortunato Fortes}

Análise e/ou interpretação dos dados, revisão final com participação crítica e intelectual no manuscrito.

\section{Como citar este artigo}

Covalski D, Pauli E, Echer AK, Nogueira RR, Fortes VLF. Postoperative of cardiac surgeries: prevalent complications within 72 hours. Rev. Enferm. UFSM. 2021 [Access on: Year Month Day]; vol.11 e75: 1-20. DOI: https://doi.org/10.5902/2179769264147 